\title{
GROWTH, GRAIN DEVELOPMENT AND YIELD PERFORMANCE OF BORO RICE VARIETIES UNDER WATER STRESS CONDITION
}

\author{
C.K. Apu', P.K. Biswas ${ }^{2 *}$, T.S. Roy ${ }^{2}$, S. Shome ${ }^{2}$ and A. Barman ${ }^{3}$ \\ ${ }^{1}$ Department of Agricultural Extension (DAE), Nasirnagar, Brahmanbaria, Bangladesh \\ ${ }^{2}$ Department of Agronomy, Sher-e-Bangla Agricultural University, Dhaka, Bangladesh \\ ${ }^{3}$ Soil Science Division, Bangladesh Agricultural Research Institute, Gazipur, Bangladesh \\ *Corresponding Email:parimalbiswas@ hotmail.com
}

(Received: 27 October 2021, Accepted: 08 November 2021)

Keywords: Irrigated rice; moisture stress; water deficit; water management; yield

\begin{abstract}
A field experiment was conducted at the Agronomy field, Sher-e-Bangla Agricultural University, Dhaka to evaluate the effect of water stress at reproductive stage on growth, grain development and yield of Boro rice. The experiment consisted of two factors: Factor A: Water stress viz. no water stress $\left(\mathrm{W}_{1}\right)$ and water stress $\left(\mathrm{W}_{2}\right)$ and Factor B: Variety viz. BRRI dhan28 $\left(\mathrm{V}_{1}\right)$, BRRI dhan29 $\left(\mathrm{V}_{2}\right)$, BRRI dhan50 $\left(\mathrm{V}_{3}\right)$, Binadhan-10 $\left(\mathrm{V}_{4}\right)$, BRRI hybriddhan $3\left(\mathrm{~V}_{5}\right)$, Aloron $\left(\mathrm{V}_{6}\right)$. In no water stress, irrigation water was maintained strictly from transplanting time to harvesting time while in water stress condition; supply of irrigation was stopped just after flowering upto harvest. Results showed that growth, grain development and yield of Boro rice were significantly affected by water stressed condition. All the tested varieties performed better under no water stress condition compared to water stress condition. About $7 \%$ panicle weight was decreased due to water stress condition at reproductive stage. Grain development of BRRI dhan29 was least affected by water stress while the most affected variety was Binadhan-10. Water deficit at reproductive stage reduced about $15-22 \%$ grain yield of tested varieties. Aloron was the least affected variety due to water stress while BRRI dhan50 was the most affected variety. The variety Aloron provided equal yield $\left(7.31 \mathrm{t} \mathrm{ha}^{-1}\right)$ under stress condition as given by BRRI dhan 29 under no water stress condition. So, Aloron may be a better option to cultivate in the region where irrigation water is scarce or costly.
\end{abstract}

\section{Introduction}

Rice which is the driving force of Bangladesh agriculture occupies about two-thirds of the cultivated land area and constitutes $90 \%$ of the food grain production in Bangladesh (BBS, 2020). Boro (January May) is the single largest crop grown in Bangladesh which accounts more than $50 \%$ of total rice production (BBS, 2020). Boro rice is generally cultivated under irrigated condition when rainfall is very scanty. Irrigated rice cultivation is the most productive and plays a vital role in fulfilling global food demand. But one estimate shows that $2000-5000 \mathrm{~L}$ of water is required to produce $1 \mathrm{~kg}$ of rice (Caine et al. 2019). But accelerated urbanization and industrialization result in decreasing freshwater resources for rice production. Therefore, time demands the production of "more rice with less water" for global food security (Maneepitak et al., 2019). Water stress is one of the most restrictive factors for growing rice (Halder et al., 2018). Keller (2005) reported that water flow through the soil-root-shoot pathway assists nutrient uptake by plant roots and leaf transpiration generates the tension necessary for the roots to absorb this essential solution so in a dry soil, uptake of water and nutrients becomes progressively more difficult for any crop. Moisture stress hampers photosynthesis in plants by closing stomata and destructing the chlorophyll contents and photosynthetic apparatus(Waraich et al., 2011).Disturbed photosynthesis adversely affects the tiller number, leaf area, dry matter production, 
filled grains per panicle, 1000-grain weight and grain yield (Sabetfar et al., 2013). Drought stress at vegetative phase of rice has minimal effect on subsequent growth and grain yield whereas drought during reproductive stage causes about $30 \%$ yield reduction (Fofana et al., 2010). This might be due to low dry matter accumulation, delayed an thesis, reduction of the number of spikelets per panicle and decrease in numbers of filled grains (Halder and Burrage, 2003). Actually water stress during reproductive stage may reduce grain mass by $20 \%$, no. of spikelet up to $60 \%$, filled grains up to $40 \%$ which contribute as a whole to reduction in yield. Farmers use groundwater for boro rice cultivation as it is available in comparison with any other facilities. But, excessive and improper use of groundwater threatened soil horizon, environment as well as groundwater availability. Exploring the ways to reduce water use for rice production is therefore of great strategic value for sustainable crop production for the world facing water scarcity (Molden et al., 2010).Considering the above fact, a detailed study was undertaken to explore the extent of water stress at reproductive stage affects growth and yield of rice and to find out suitable varieties providing high yield under water stress condition and saving the cost of irrigation water in boro rice production.

\section{Materials and Methods}

The experiment was conducted at Sher-e-Bangla Agricultural University $\left(23^{\circ} 77^{\prime} \mathrm{N}\right.$ latitude and $90^{\circ} 33^{\prime} \mathrm{E}$ longitude) which belongs to the Agro-ecological zone of The Modhupur Tract, AEZ-28 (Anonymous, 1988). Split plot design with three replications was followed to implement the experiment where water stress was put in the mainplots and variety in the sub-plots. There were two water stress conditions $\left(\mathrm{W}_{1}\right.$ : no water stress and $\mathrm{W}_{2}$ : water stress) and six varieties $\mathrm{V}_{1}$ : BRRI dhan28, $\mathrm{V}_{2}$ : BRRI dhan29, $\mathrm{V}_{3}$ : BRRI dhan50, $\mathrm{V}_{4}$ : Binadhan-10, $\mathrm{V}_{5}$ : BRRI hybriddhan3 and $\mathrm{V}_{6}$ : Aloron. Water stress means absence of irrigation water from reproductive stage to harvesting stage where no water stage means no scarcity of irrigation water during rice cultivation. Before reproductive stage there is no discrimination of irrigation water among the plots. Unit plot of $4 \mathrm{~m} \times 2 \mathrm{~m}$ was made with $0.75 \mathrm{~m}$ distance between plot to plot.Thirtydays old seedlings were transplanted in the main field. The experimental area was fertilized with 120, 80, 80, 20 and $5 \mathrm{~kg} \mathrm{ha}^{-1}$ of $\mathrm{N}, \mathrm{P}_{2} \mathrm{O}_{5}, \mathrm{~K}_{2} \mathrm{O}, \mathrm{S}$ and $\mathrm{Zn}$ as per recommended method (FRG, 2012). Crop management was practiced according to necessity. Plant height, number of tillers hiil $^{-1}$ and number of leaves hiil ${ }^{-1}$ were calculated from randomly pre-selected 5 hills plot ${ }^{-1}$. Dry matter was recorded from the mean oven dry weight of plants from 2 hills per plot. Filled grains panicle ${ }^{-1}$ and unfilled grains panicle ${ }^{-1}$ were counted from the average number of grains from ten panicles.1000-grain weight was measured at $12 \%$ moisture content.Grain yield and straw yield were determined from the central $4 \mathrm{~m}^{2}$ area of each plot and expressed as $\mathrm{t} \mathrm{ha}^{-1}$. Biological yield was calculated by summing up the grain and straw yield. Harvest index refers to the ratio of economic yield to biological yield and was computed with following formula (Gardner et al., 1985).

$$
\text { Harvest index }(\%)=\frac{\text { Grain yield }}{\text { Biological yield }} \times 100
$$

All the data collected on different parameters were statistically analyzed following the analysis of variance (ANOVA) technique using STATISTIX 10 computer package program and the mean differences were calculated using least significant difference (LSD) test at $5 \%$ level of significance.

\section{Results and Discussion}

\section{Effect of water stress on crop growth characters}

Crop growth parameters viz. plant height, number of tillers hill-1 and number of leaves hill $^{-1}$ showed significant variation when water stress was imposed at reproductive stage (Table 1). Plants under no water stress condition were found to be a bit taller with higher number of tillers and leaves than the plants under water stress condition. Number of tillers and leaves hill ${ }^{-1}$ were reduced by about $8 \%$ and $12 \%$, respectively due to water stress condition. About $12 \%$ higher stem dry weight was recorded for 
no water stress condition than water stress condition. On the other hand, leaf dry weight and root dry weight were not influenced by water stress condition. Sokoto and Muhammad (2014)found water stress at flowering and grain filling stage decreased plant height. Plant cannot uptake sufficient nutrients from soil due to water scarcity which consequently caused plant growth retardation (Mannan et al., 2012). Mostajeran and Rahimi-Eichi (2009) documented decreased tiller number per hill due to decreased soil moisture level. Inability to produce dry matter due to inhibited photosynthesis by moisture stress might be responsible for lower tiller production. Dearth of water required for food production and cell division might be another reason (Zubaer et al., 2007).

\section{Effect of variety on crop growth characters}

Varietal differences had significant impact on all growth parameters (Table 1). At harvest, tallest plant $(106.98 \mathrm{~cm})$ was recorded from Binadhan-10 and shortest plant $(85.03 \mathrm{~cm})$ was obtained from BRRI dhan50. Maximum number of tillers (14.88) and leaves (48.64) were observed in BRRI dhan50 while minimum tillers (10.50) and leaves (23.0) were recorded from Aloron. BRRI dhan50 provided lowest leaf dry weight while Binadhan-10 produced lowest stem and root dry weight at harvest. The variety BRRIdhan29 gave highest stem and root dry weight which was significantly different from other varieties. The variation in plant height among the different varieties might be due to genetic makeup of different varieties (Murshida et al., 2017).

Table 1. Effect of water stress and variety on growth parameters of Boro rice at harvest

\begin{tabular}{|c|c|c|c|c|c|c|}
\hline Treatments & $\begin{array}{l}\text { Plant height } \\
\text { (cm) }\end{array}$ & $\begin{array}{l}\text { Number of } \\
\text { tillers hill - } \\
\quad 1\end{array}$ & $\begin{array}{c}\text { Number of } \\
\text { leaves } \\
\text { hill }^{-1}\end{array}$ & $\begin{array}{c}\text { Leaf dry } \\
\text { weight } \\
\left(\mathrm{g} \text { hill }^{-1}\right)\end{array}$ & $\begin{array}{c}\text { Stem dry } \\
\text { weight } \\
\left.\text { (g hill }^{-1}\right)\end{array}$ & $\begin{array}{c}\text { Root dry } \\
\text { weight } \\
\left.\text { (g hill }^{-1}\right)\end{array}$ \\
\hline \multicolumn{7}{|l|}{ Water stress } \\
\hline $\mathrm{W}_{1}$ & $98.35 \mathrm{a}$ & $12.48 \mathrm{a}$ & $35.05 \mathrm{a}$ & 16.77 & $31.31 \mathrm{a}$ & 9.39 \\
\hline $\mathbf{W}_{2}$ & $93.68 \mathrm{~b}$ & $11.53 \mathrm{~b}$ & $30.79 \mathrm{~b}$ & 15.05 & $27.57 \mathrm{~b}$ & 8.23 \\
\hline $\operatorname{LSD}_{(0.05)}$ & 4.27 & 0.60 & 2.36 & NS & 2.29 & NS \\
\hline $\mathrm{CV}(\%)$ & 3.09 & 23.82 & 12.07 & 13.76 & 5.41 & 21.02 \\
\hline \multicolumn{7}{|l|}{ Variety } \\
\hline$\dot{V}_{1}$ & $94.75 \mathrm{~b}$ & $12.35 \mathrm{ab}$ & $42.81 \mathrm{~b}$ & $19.04 \mathrm{a}$ & $33.24 \mathrm{ab}$ & $9.67 \mathrm{ab}$ \\
\hline $\mathbf{V}_{\mathbf{2}}$ & $95.46 \mathrm{~b}$ & $12.47 \mathrm{ab}$ & $25.16 \mathrm{~d}$ & $19.51 \mathrm{a}$ & $35.89 \mathrm{a}$ & $12.04 \mathrm{a}$ \\
\hline$V_{3}$ & $85.03 \mathrm{c}$ & $14.87 \mathrm{a}$ & $48.64 \mathrm{a}$ & $9.45 \mathrm{c}$ & $26.40 \mathrm{c}$ & $7.21 \mathrm{bc}$ \\
\hline$V_{4}$ & $106.98 \mathrm{a}$ & $10.93 \mathrm{~b}$ & $32.93 \mathrm{c}$ & $16.06 \mathrm{a}$ & $26.04 \mathrm{c}$ & $6.27 \mathrm{c}$ \\
\hline$V_{5}$ & $98.29 \mathrm{~b}$ & $10.90 \mathrm{~b}$ & $25.00 \mathrm{~d}$ & $14.30 \mathrm{~b}$ & $27.06 \mathrm{c}$ & $8.03 \mathrm{bc}$ \\
\hline$V_{6}$ & $95.56 \mathrm{~b}$ & $10.50 \mathrm{~b}$ & $23.00 \mathrm{~d}$ & $17.09 \mathrm{ab}$ & $27.99 \mathrm{bc}$ & $9.66 \mathrm{ab}$ \\
\hline $\operatorname{LSD}_{(0.05)}$ & 3.54 & 2.74 & 5.80 & 4.18 & 5.79 & 2.51 \\
\hline CV (\%) & 3.06 & 17.92 & 14.63 & 21.81 & 16.34 & 23.63 \\
\hline
\end{tabular}

$\mathrm{W}_{1}$ - No water stress, $\mathrm{W}_{2}$ - Water stress and $\mathrm{V}_{1}$ - BRRI dhan28, $\mathrm{V}_{2}$ - BRRI dhan29, $\mathrm{V}_{3}$ - BRRI dhan50,

$\mathrm{V}_{4}$ - Binadhan-10, $\mathrm{V}_{5}$ - BRRI hybrid dhan3, $\mathrm{V}_{6}$ - Aloron.

\section{Interaction effect of water stress and varietyon crop growth characters}

Interaction effect of water stress and variety had significant influence on all crop growth parameters (Table 2). All the tested varieties performed better under no water stress condition. When there was no water stress throughout the crop duration, tallest plant $(110.37 \mathrm{~cm})$ was recorded from Binadhan-10 and maximum number of tillers and leaves were recorded from BRRI dhan50. At harvest, the variety BRRI dhan 29 provided maximum leaf, root and stem dry weight. When water supply was stopped at reproductive stage, growth and development of all the tested varieties were hampered considerably. Tiller and leaf production of BRRI dhan50 was least affected by water stress. BRRI dhan29 and Aloron accumulated more dry matter in stem, leaves and root at harvest under water stress condition compared to other varieties. Chowdhury et al. (2004) observed that plant height decreased with the decrease in soil moisture levels and elucidated inhibition of cell division and cell enlargement due to water stress as the possible reason. 
Table 2. Interaction effect of water stress and variety on growth parameters of Boro rice at harvest

\begin{tabular}{|c|c|c|c|c|c|c|}
\hline Treatments & $\begin{array}{c}\text { Plant } \\
\text { height }(\mathbf{c m})\end{array}$ & $\begin{array}{l}\text { Number of } \\
\text { tillers hill }^{-1}\end{array}$ & $\begin{array}{c}\text { Number of } \\
\text { leaves } \\
\text { hill }^{-1}\end{array}$ & $\begin{array}{c}\text { Dry Leaf } \\
\text { weight } \\
\left.\text { (g hill- }^{-1}\right)\end{array}$ & $\begin{array}{c}\text { Dry stem } \\
\text { weight } \\
\left.\text { (g hill }^{-1}\right)\end{array}$ & $\begin{array}{c}\text { Dry Root } \\
\text { weight } \\
\left.\text { (g hill }^{-1}\right)\end{array}$ \\
\hline $\mathrm{W}_{1} \mathrm{~V}_{1}$ & $97.25 \mathrm{~cd}$ & $12.60 \mathrm{abc}$ & $46.13 \mathrm{ab}$ & $19.74 \mathrm{a}$ & $35.89 \mathrm{ab}$ & $10.12 \mathrm{ab}$ \\
\hline $\mathrm{W}_{1} \mathrm{~V}_{2}$ & $97.41 \mathrm{~cd}$ & $13.80 \mathrm{ab}$ & $28.07 \mathrm{def}$ & $20.96 \mathrm{a}$ & $39.93 \mathrm{a}$ & $13.34 \mathrm{a}$ \\
\hline $\mathrm{W}_{1} \mathrm{~V}_{3}$ & 86.68 ef & $15.61 \mathrm{a}$ & $51.75 \mathrm{a}$ & $11.49 \mathrm{bc}$ & $27.86 \mathrm{bcd}$ & $9.13 \mathrm{bc}$ \\
\hline $\mathrm{W}_{1} \mathrm{~V}_{4}$ & $110.37 \mathrm{a}$ & $11.07 \mathrm{bc}$ & $34.20 \mathrm{~cd}$ & $16.27 \mathrm{ab}$ & $25.35 \mathrm{~cd}$ & $5.98 \mathrm{~cd}$ \\
\hline $\mathrm{W}_{1} \mathrm{~V}_{5}$ & $101.17 \mathrm{bc}$ & $11.00 \mathrm{bc}$ & $25.47 \mathrm{ef}$ & $16.91 \mathrm{ab}$ & $31.14 \mathrm{bc}$ & $8.52 \mathrm{bcd}$ \\
\hline $\mathrm{W}_{1} \mathrm{~V}_{6}$ & $97.21 \mathrm{~cd}$ & $10.80 \mathrm{bc}$ & 24.73 ef & $15.24 \mathrm{ab}$ & $27.67 \mathrm{~cd}$ & $9.26 b c$ \\
\hline $\mathrm{W}_{2} \mathrm{~V}_{1}$ & $92.25 \mathrm{de}$ & $12.10 \mathrm{abc}$ & $39.50 \mathrm{bc}$ & $18.35 \mathrm{a}$ & $30.58 \mathrm{bcd}$ & $9.22 \mathrm{bc}$ \\
\hline $\mathrm{W}_{2} \mathrm{~V}_{2}$ & $93.51 \mathrm{~d}$ & $11.13 \mathrm{bc}$ & $22.27 \mathrm{f}$ & $18.06 \mathrm{a}$ & $31.86 \mathrm{bc}$ & $10.74 \mathrm{ab}$ \\
\hline $\mathrm{W}_{2} \mathrm{~V}_{3}$ & $83.39 \mathrm{f}$ & $14.13 \mathrm{ab}$ & $45.53 \mathrm{ab}$ & $7.41 \mathrm{c}$ & $24.93 \mathrm{~cd}$ & $5.28 \mathrm{~d}$ \\
\hline $\mathrm{W}_{2} \mathrm{~V}_{4}$ & $103.60 \mathrm{~b}$ & $10.80 \mathrm{bc}$ & $31.67 \mathrm{cde}$ & $15.85 \mathrm{ab}$ & $26.74 \mathrm{~cd}$ & $6.56 \mathrm{~cd}$ \\
\hline $\mathrm{W}_{2} \mathrm{~V}_{5}$ & $95.41 \mathrm{~cd}$ & $10.80 \mathrm{bc}$ & 24.53 ef & $11.69 \mathrm{bc}$ & $22.97 \mathrm{~d}$ & $7.53 \mathrm{bcd}$ \\
\hline $\mathrm{W}_{2} \mathrm{~V}_{6}$ & $93.91 \mathrm{~d}$ & $10.20 \mathrm{c}$ & $21.27 \mathrm{f}$ & $18.95 \mathrm{a}$ & $28.32 \mathrm{bcd}$ & $10.05 \mathrm{ab}$ \\
\hline $\operatorname{LSD}_{(0.05)}$ & 5.009 & 3.790 & 8.20 & 5.910 & 8.190 & 3.490 \\
\hline $\mathrm{CV}(\%)$ & 3.06 & 17.92 & 14.63 & 21.81 & 16.34 & 23.63 \\
\hline
\end{tabular}

$\mathrm{W}_{1}$ - No water stress, $\mathrm{W}_{2}$ - Water stress and $\mathrm{V}_{1}$ - BRRI dhan28, $\mathrm{V}_{2}$ - BRRI dhan29, $\mathrm{V}_{3}$ - BRRI dhan50,

$\mathrm{V}_{4}$ - Binadhan-10, $\mathrm{V}_{5}$ - BRRI hybrid dhan3, $\mathrm{V}_{6}$ - Aloron.

\section{Effect of water stress on yield and yield contributing characters}

Filled grainspanicle ${ }^{-1}$, 1000-grains weight and harvest index were not significantly influenced by different water stress condition while water stress had significant effect on unfilled grainspanicle ${ }^{-1}$, grain yield, straw yield and biological yield (Table 3 ). All the yield and yield contributing characters achieved maximum value under no water stress condition. Water stress at reproductive stages caused about $14 \%$ yield reduction compared to no water stress condition. Mannan et al. (2012)reported lower number of filled grains per panicle and grain weight due to water stress at reproductive stage. Inactivation of pollen grain due to dryness, hampered pollen tube development and disturbed assimilates production and distribution might be the causes (Fofana et al., 2010).Actually moisture stress obstructs photosynthesis and limits the supply of photosynthates to developing grains which eventually reduces grain yield.

\section{Effect of variety on yield and yield contributing characters}

Varietal performance had significant impact on yield and yield contributing characters (Table 3). The maximum number of filled grains panicle ${ }^{-1}(133.28)$ was obtained from BRRI dhan29 which was statistically at par with BRRI dhan28 and BRRI hybrid dhan3. The lowest number of filled grains panicle $^{-1}$ (98.88) was recorded from Aloron. Maximum 1000-grain weight (26.55 g) was obtained from BRRI hybrid dhan 3 and minimum $(21.03 \mathrm{~g})$ from BRRI dhan28. Aloron provided maximum grain yield which was $12 \%$ and $18 \%$ higher than BRRI hybrid dhan3 and BRRI dhan29. Lowest yielder BRRI dhan50 and BRRI dhan28 gave about 50\% lower yield than Aloron. Aloron also had the highest straw yield and biological yield. Maximum harvest index (49.64\%) was recorded from BRRI dhan29 which was statistically similar with other varieties except BRRI dhan50 and BRRI hybrid dhan3. Mannan et al. (2012) also documented significant variations in case of yield and yield contributing attributes due to difference in genetic makeup of varieties.

Table 3. Effect of water stress and variety on yield and yield contributing characters of Boro rice

\begin{tabular}{cccccccc}
\hline $\begin{array}{c}\text { Treat- } \\
\text { ments }\end{array}$ & $\begin{array}{c}\text { Filled grains } \\
\text { panicle }^{-1}\end{array}$ & $\begin{array}{c}\text { Unfilled } \\
\text { grains } \\
\text { panicle }\end{array}$ & $\begin{array}{c}\text { 1000- grain } \\
\text { weight }\end{array}$ & $\begin{array}{c}\text { Grain yield } \\
\left(\mathbf{t ~ h a}^{-1}\right)\end{array}$ & $\begin{array}{c}\text { Straw yield } \\
\left(\mathrm{t} \mathrm{ha}^{-1}\right)\end{array}$ & $\begin{array}{c}\text { Biolo- } \\
\text { gical yield } \\
\left(\mathbf{t ~ h a}^{-1}\right)\end{array}$ & $\begin{array}{c}\text { Harvest } \\
\text { index } \\
(\%)\end{array}$ \\
\hline
\end{tabular}




\begin{tabular}{cccccccc}
\hline Water stress & & & & & & \\
$\mathrm{W}_{1}$ & 117.8 & 14.23 & 22.31 & $6.86 \mathrm{a}$ & $7.4 \mathrm{a}$ & $14.26 \mathrm{a}$ & 48.1 \\
$\mathrm{~W}_{2}$ & 106.6 & 20.04 & 22.26 & $5.75 \mathrm{~b}$ & $6.47 \mathrm{~b}$ & $12.22 \mathrm{~b}$ & 47 \\
\hline $\mathrm{LSD}_{(0.05)}$ & $\mathrm{NS}$ & 1.87 & $\mathrm{NS}$ & 0.48 & 0.79 & 1.24 & $\mathrm{NS}$ \\
$\mathrm{CV}(\%)$ & 8.14 & 7.59 & 0.54 & 5.31 & 7.91 & 1.95 & 1.95 \\
\hline Variety & & & & & & & \\
$\mathrm{V}_{1}$ & $121.25 \mathrm{a}$ & $21.27 \mathrm{a}$ & $21.03 \mathrm{c}$ & $5.14 \mathrm{~d}$ & $5.70 \mathrm{~d}$ & $10.84 \mathrm{~d}$ & $47.35 \mathrm{ab}$ \\
$\mathrm{V}_{2}$ & $133.28 \mathrm{a}$ & $20.70 \mathrm{a}$ & $18.37 \mathrm{~d}$ & $6.74 \mathrm{~b}$ & $6.84 \mathrm{~b}$ & $13.57 \mathrm{c}$ & $49.64 \mathrm{a}$ \\
$\mathrm{V}_{3}$ & $102.15 \mathrm{bc}$ & $21.42 \mathrm{a}$ & $18.37 \mathrm{~d}$ & $5.05 \mathrm{~d}$ & $5.91 \mathrm{~d}$ & $10.97 \mathrm{~d}$ & $46.17 \mathrm{~b}$ \\
$\mathrm{~V}_{4}$ & $100.82 \mathrm{bc}$ & $10.48 \mathrm{~b}$ & $25.19 \mathrm{~b}$ & $5.85 \mathrm{c}$ & $6.55 \mathrm{bc}$ & $12.40 \mathrm{c}$ & $47.20 \mathrm{ab}$ \\
$\mathrm{V}_{5}$ & $117.17 \mathrm{ab}$ & $11.95 \mathrm{~b}$ & $26.55 \mathrm{a}$ & $7.08 \mathrm{~b}$ & $8.09 \mathrm{a}$ & $15.17 \mathrm{~b}$ & $46.68 \mathrm{~b}$ \\
$\mathrm{~V}_{6}$ & $98.88 \mathrm{c}$ & $17.00 \mathrm{ab}$ & $25.40 \mathrm{~b}$ & $7.98 \mathrm{a}$ & $8.53 \mathrm{a}$ & $16.51 \mathrm{a}$ & $48.25 \mathrm{ab}$ \\
\hline $\mathrm{LSD}_{(0.05)}$ & 16.4 & 7.78 & 0.66 & 0.63 & 0.75 & 1.18 & 2.87 \\
$\mathrm{CV}(\%)$ & 12.13 & 25.01 & 2.45 & 8.31 & 8.94 & 7.42 & 5.00 \\
\hline
\end{tabular}

$\mathrm{W}_{1}$ - No water stress, $\mathrm{W}_{2}$ - Water stress and $\mathrm{V}_{1}$ - BRRI dhan28, $\mathrm{V}_{2}$ - BRRI dhan29, V3- BRRI dhan50,

V4- Binadhan-10, V5- BRRI hybrid dhan3, V6- Aloron.

\section{Interaction effect of water stress and varietyon yield and yield contributing characters}

The interaction between water stress and variety significantly influenced yield and yield contributing characters (Table 4). All the tested varieties performed better under no water stress condition than water stress condition from reproductive stage to harvest. BRRI dhan29 gave the maximum filled grainspanicle ${ }^{-1}$ both under no water stress condition (145.43) and water stress condition(121.13). Number of unfilled grains was found minimum in Binadhan-10 under both the situation when plants faced no water stress and water stress at reproductive stage. Grain yield was considerably reduced (15$22 \%$ ) due to water stress condition in case of all the varieties. Aloron provided maximum grain yield in both the cases of no water stress and water stress but water stress reduced about $15 \%$ yield of that variety. However, grain yield of Aloron was the least affected due to water stress while BRRI dhan50 (22\% yield reduction) was the most affected variety by water stress. Better performance was also recorded from Aloron in case of straw yield and biological yield. Harvest index of varieties was not significantly influenced by water stress except BRRI dhan50. Sarvestani et al. (2008) documented that water stress at reproductive stage reduced about $50 \%$ grain yield in comparison to no water stress condition.

Table 4. Interaction effect of water stress and variety on yield and yield contributing characters of Boro rice

\begin{tabular}{|c|c|c|c|c|c|c|c|}
\hline Treatments & $\begin{array}{l}\text { Filled grains } \\
\text { panicle }^{-1}(\text { No. })\end{array}$ & $\begin{array}{c}\text { Unfilled } \\
\text { grains } \\
\text { panicle }^{-1}\end{array}$ & $\begin{array}{c}\text { 1000- } \\
\text { grain } \\
\text { weight }(\mathrm{g})\end{array}$ & $\begin{array}{l}\text { Grain } \\
\text { yield } \\
\left(\mathrm{t} \mathrm{ha}^{-1}\right)\end{array}$ & $\begin{array}{c}\text { Straw } \\
\text { yield } \\
\left(\mathrm{t} \mathrm{ha}^{-1}\right)\end{array}$ & $\begin{array}{c}\text { Biolo-gical } \\
\text { yield } \\
\left(\mathrm{t} \mathrm{ha}^{-1}\right)\end{array}$ & $\begin{array}{c}\text { Harvest } \\
\text { index } \\
(\%)\end{array}$ \\
\hline $\mathrm{W}_{1} \mathrm{~V}_{1}$ & $123.63 \mathrm{abc}$ & $16.40 \mathrm{abc}$ & $21.13 \mathrm{~d}$ & $5.63 \mathrm{def}$ & $6.10 \mathrm{~d}$ & $11.72 \mathrm{ef}$ & $48.01 \mathrm{a}$ \\
\hline $\mathrm{W}_{1} \mathrm{~V}_{2}$ & $145.43 \mathrm{a}$ & $16.47 \mathrm{abc}$ & $18.26 \mathrm{e}$ & $7.31 \mathrm{bc}$ & $7.44 \mathrm{~b}$ & $14.76 \mathrm{bc}$ & $49.55 \mathrm{a}$ \\
\hline $\mathrm{W}_{1} \mathrm{~V}_{3}$ & $100.07 \mathrm{de}$ & $16.70 \mathrm{abc}$ & $17.23 \mathrm{f}$ & 5.59 ef & $5.93 \mathrm{~d}$ & 11.52 ef & $48.68 \mathrm{a}$ \\
\hline $\mathrm{W}_{1} \mathrm{~V}_{4}$ & $102.73 \mathrm{cde}$ & $8.40 \mathrm{c}$ & $25.37 \mathrm{bc}$ & $6.33 \mathrm{de}$ & $7.30 \mathrm{bc}$ & $13.63 \mathrm{~cd}$ & $46.46 \mathrm{ab}$ \\
\hline $\mathrm{W}_{1} \mathrm{~V}_{5}$ & $126.57 \mathrm{ab}$ & $9.437 \mathrm{c}$ & $26.10 \mathrm{~b}$ & $7.65 \mathrm{~b}$ & $8.73 \mathrm{a}$ & $16.39 \mathrm{ab}$ & $46.69 \mathrm{ab}$ \\
\hline $\mathrm{W}_{1} \mathrm{~V}_{6}$ & $108.67 \mathrm{~b}-\mathrm{e}$ & $14.97 \mathrm{abc}$ & $25.47 \mathrm{bc}$ & $8.64 \mathrm{a}$ & $8.91 \mathrm{a}$ & $17.55 \mathrm{a}$ & $49.19 \mathrm{a}$ \\
\hline $\mathrm{W}_{2} \mathrm{~V}_{1}$ & $118.87 \mathrm{bcd}$ & $26.13 \mathrm{a}$ & $25.47 \mathrm{bc}$ & $4.65 \mathrm{~g}$ & $5.30 \mathrm{~d}$ & $9.95 \mathrm{f}$ & $46.68 \mathrm{ab}$ \\
\hline $\mathrm{W}_{2} \mathrm{~V}_{2}$ & $121.13 \mathrm{bcd}$ & $21.93 \mathrm{ab}$ & $18.49 \mathrm{e}$ & $6.16 \mathrm{def}$ & $6.23 \mathrm{~cd}$ & $12.39 \mathrm{de}$ & $49.72 \mathrm{a}$ \\
\hline $\mathrm{W}_{2} \mathrm{~V}_{3}$ & $104.23 \mathrm{~b}-\mathrm{e}$ & $26.13 \mathrm{a}$ & $17.10 \mathrm{f}$ & $4.52 \mathrm{~g}$ & $5.90 \mathrm{~d}$ & $10.42 \mathrm{f}$ & $43.66 \mathrm{~b}$ \\
\hline $\mathrm{W}_{2} \mathrm{~V}_{4}$ & $98.90 \mathrm{de}$ & $12.57 \mathrm{bc}$ & $25.01 \mathrm{c}$ & $5.36 \mathrm{fg}$ & $5.80 \mathrm{~d}$ & 11.17 ef & $47.94 \mathrm{a}$ \\
\hline $\mathrm{W}_{2} \mathrm{~V}_{5}$ & $107.77 \mathrm{~b}-\mathrm{e}$ & $14.47 \mathrm{abc}$ & $27.00 \mathrm{a}$ & $6.52 \mathrm{~cd}$ & $7.45 \mathrm{~b}$ & $13.96 \mathrm{~cd}$ & $46.67 \mathrm{ab}$ \\
\hline $\mathrm{W}_{2} \mathrm{~V}_{6}$ & $89.10 \mathrm{e}$ & $19.03 \mathrm{abc}$ & $25.33 \mathrm{bc}$ & $7.31 \mathrm{bc}$ & $8.15 \mathrm{ab}$ & $15.47 \mathrm{bc}$ & $47.32 \mathrm{ab}$ \\
\hline $\operatorname{LSD}_{(0.05)}$ & 23.19 & 12.86 & 0.93 & 0.89 & 1.06 & 1.63 & 4.05 \\
\hline CV (\%) & 12.13 & 25.01 & 2.45 & 8.31 & 8.94 & 7.42 & 5.00 \\
\hline
\end{tabular}

$\mathrm{W}_{1}$ - Nowater stress, $\mathrm{W}_{2}$ - Water stress and $\mathrm{V}_{1}$ - BRRI dhan28, V2- BRRI dhan29, V3- BRRI dhan50, V4- Binadhan-10, V5BRRI hybrid dhan3, $\mathrm{V}_{6-}$ Aloron. 


\section{Effect of water stress on grain growth pattern}

Panicle weight was significantly influenced by water stress at 5, 10, 15, 20, 25 days after anthesis and at harvest (Figure 1). Panicle weight increased from 5 days after anthesis up to harvest for both no water stress and water stress condition. Panicle weight was reduced by about $7 \%$ at harvest due to water stress condition at reproductive stage. Fofana et al.(2010)found that effect of water stress on yield was most severe when drought occurred during panicle development. This might be associated with low dry matter production during the drought period as well as during the recovery period following the drought. When drought occurred during grain filling, the percentage of filled grain was decreased to $40 \%$ and individual grain mass decreased by $20 \%$ (Sarvestani et al., 2008). Mannan et al. (2012) also found variation in grain development due to water deficit condition.

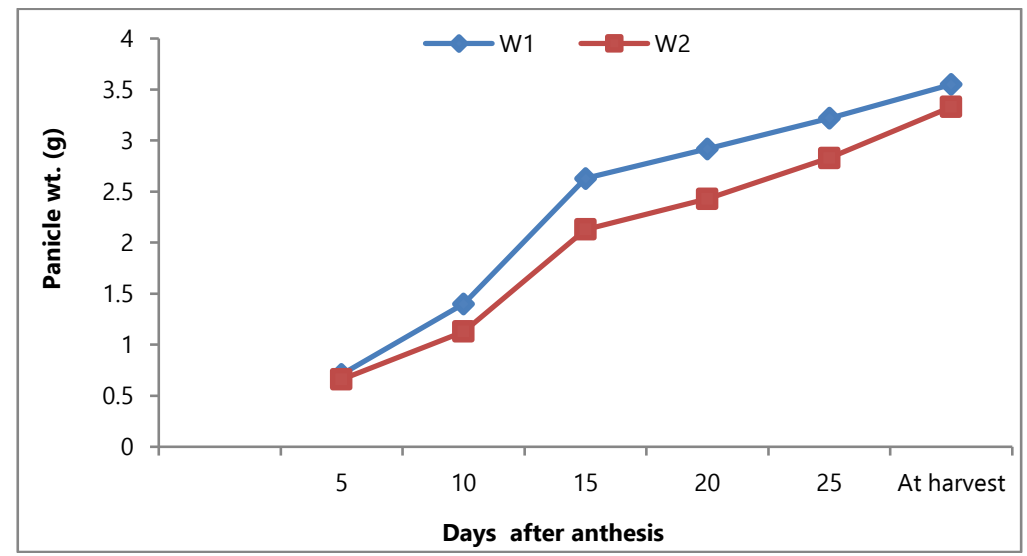

$\mathrm{W}_{1}$-No water stress, $\mathrm{W}_{2}$-Water stress

$\left(\operatorname{LSD}_{(0.05)}\right.$ value $=0.06,0.33,0.23,0.29,0.24$ and 0.11 at $5,10,15,20,25$ days after anthesis and at harvest, respectively).

Fig.1. Effect of water stress on panicle weight at different days after anthesis

\section{Effect of variety on grain growth pattern}

Varietal variances showed significant difference in anthesis development. Panicle weight was significantly influenced by different varieties at 15, 25 days after anthesis and at harvest but statistically similar at 5 and 10 days after anthesis (Figure 2). Panicle weight of all the tested varieties was increased throughout the grain development period with a little fluctuation. At harvest, maximum panicle weight was recorded in BRRI hybrid dhan3 (4.61 g) which was statistically similar with Aloron $(4.42 \mathrm{~g})$. On the other hand, BRRI dhan50produced the minimum panicle weight at harvest (2.30 g).These findings corroborated with the findings of Murshida et al. (2017).

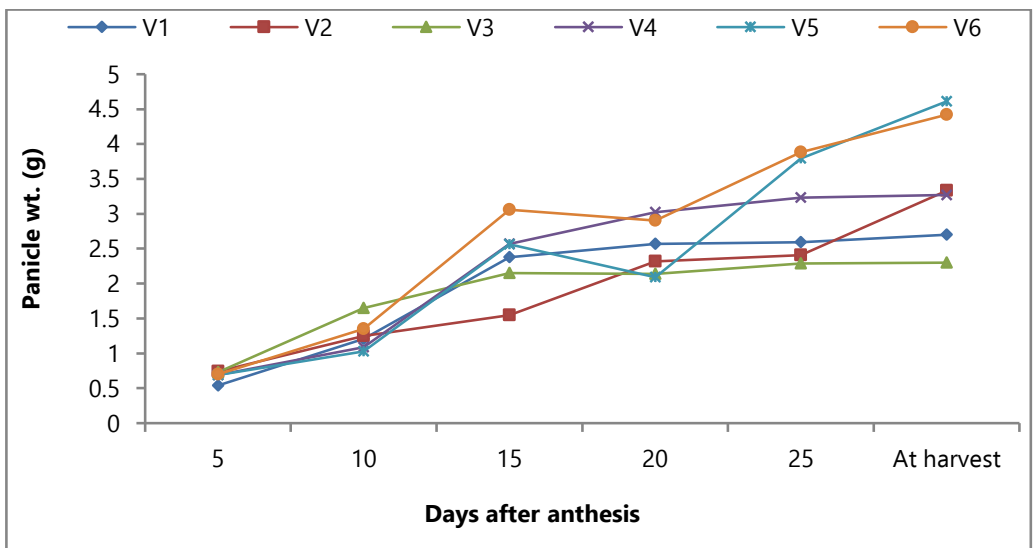


$\mathrm{V}_{1}$ - BRRI dhan28, $\mathrm{V}_{2}$ - BRRI dhan29, $\mathrm{V}_{3}$ - BRRI dhan50, $\mathrm{V}_{4}$ - Binadhan-10, $\mathrm{V}_{5}$ - BRRI hybriddhan3, $\mathrm{V}_{6}-$ Aloron $\left(\operatorname{LSD}_{(0.05)}=\mathrm{NS}, \mathrm{NS}, 0.61,0.56,0.46\right.$ and 0.75 at 5, 10, 15, 20, 25 days after anthesis and at harvest, respectively).

Fig. 2. Effect of variety on Panicle weight at different days after anthesis

\section{Interaction effect of water stress and varietyon grain growth pattern}

Panicle weight was significantly influenced by the interaction of water stress and variety at 15, 20, 25 days after anthesis and at harvest but statistically insignificantat 5 and 10 days after anthesis (Table 5).

Table 5. Interaction effect of water stress and variety on panicle weight at different days after anthesis

\begin{tabular}{|c|c|c|c|c|c|c|}
\hline Treatments & 5 Days & 10 Days & 15 Days & 20 Days & 25 Days & At harvest \\
\hline $\mathrm{W}_{1} \mathrm{~V}_{1}$ & 1.3 & 1.45 & $2.70 \mathrm{a}-\mathrm{d}$ & $2.74 \mathrm{a}-\mathrm{e}$ & $2.76 \mathrm{~cd}$ & $2.78 \mathrm{~d}$ \\
\hline $\mathrm{W}_{1} \mathrm{~V}_{2}$ & 0.80 & 1.33 & $1.73 \mathrm{de}$ & $2.78 \mathrm{a}-\mathrm{e}$ & $2.73 \mathrm{~cd}$ & $3.34 \mathrm{bcd}$ \\
\hline $\mathrm{W}_{1} \mathrm{~V}_{3}$ & 0.73 & 2.00 & $2.31 \mathrm{a}-\mathrm{e}$ & $2.25 \mathrm{def}$ & 2.38 cde & $2.39 \mathrm{~d}$ \\
\hline $\mathrm{W}_{1} \mathrm{~V}_{4}$ & 0.72 & 1.04 & $3.04 \mathrm{ab}$ & $3.40 \mathrm{a}$ & $3.50 \mathrm{ab}$ & $3.52 \mathrm{a}-\mathrm{d}$ \\
\hline $\mathrm{W}_{1} \mathrm{~V}_{5}$ & 0.71 & 1.10 & $2.77 \mathrm{abc}$ & $3.29 \mathrm{ab}$ & $4.01 \mathrm{a}$ & $4.84 \mathrm{a}$ \\
\hline $\mathrm{W}_{1} \mathrm{~V}_{6}$ & 0.74 & 1.22 & $3.20 \mathrm{a}$ & $3.08 \mathrm{abc}$ & $3.95 \mathrm{a}$ & $4.46 \mathrm{ab}$ \\
\hline $\mathrm{W}_{2} \mathrm{~V}_{1}$ & 0.52 & 0.97 & $2.05 \mathrm{~b}-\mathrm{e}$ & $2.40 \mathrm{c}-\mathrm{f}$ & 2.41 cde & $2.63 \mathrm{~d}$ \\
\hline $\mathrm{W}_{2} \mathrm{~V}_{2}$ & 0.68 & 1.17 & $1.36 \mathrm{e}$ & $1.88 \mathrm{f}$ & $2.08 \mathrm{e}$ & $3.32 \mathrm{bcd}$ \\
\hline $\mathrm{W}_{2} \mathrm{~V}_{3}$ & 0.73 & 1.30 & $1.98 \mathrm{cde}$ & $2.047 \mathrm{ef}$ & $2.19 \mathrm{de}$ & $2.32 \mathrm{bcd}$ \\
\hline $\mathrm{W}_{2} \mathrm{~V}_{4}$ & 0.66 & 1.13 & $2.10 \mathrm{~b}-\mathrm{e}$ & $2.64 b-f$ & $2.96 \mathrm{bc}$ & $3.03 \mathrm{~cd}$ \\
\hline $\mathrm{W}_{2} \mathrm{~V}_{5}$ & 0.67 & 0.96 & $2.35 \mathrm{a}-\mathrm{e}$ & $2.89 \mathrm{a}-\mathrm{d}$ & $3.56 \mathrm{ab}$ & $4.39 \mathrm{abc}$ \\
\hline $\mathrm{W}_{2} \mathrm{~V}_{6}$ & 0.66 & 1.47 & $2.91 \mathrm{abc}$ & $2.71 \mathrm{a}-\mathrm{e}$ & $3.80 \mathrm{a}$ & $4.38 \mathrm{abc}$ \\
\hline $\operatorname{LSD}_{(0.05)}$ & NS & NS & 1.00 & 0.79 & 0.65 & 1.41 \\
\hline $\mathrm{CV}(\%)$ & 16.49 & 28.80 & 14.05 & 17.34 & 12.54 & 13.67 \\
\hline
\end{tabular}

$\mathrm{W}_{1}$ - No water stress, $\mathrm{W}_{2}$ - Water stress and $\mathrm{V}_{1}$ - BRRI dhan28, V2- BRRI dhan29, V3- BRRI dhan50, V4- Binadhan-10, V5BRRI hybriddhan3, $\mathrm{V}_{6}$ - Aloron.

Panicle weight of all the tested varieties was reduced due to water stress at reproductive stage. Grain development of BRRI dhan29 was least affected by water stress while the most affected variety was Binadhan-10. Panicle weight of Binadhan-10 at harvest was declined by $14 \%$ due to water stress. Sokoto and Muhammad(2014)reported that applied moisture stress during reproduction phase reduced yield with the magnitude of yield reduction being $25.8 \%$ (average of reproduction in booting, flowering and grain filling stage) compared to control. Fofana et al. (2010)alsoshowed that stress at booting and flowering stages had the similar effect on grain yield.

\section{Conclusion}

Considering the above results, it may be concluded that water stress at reproductive stage significantly reduces the grain yield. Among the tested varieties, Aloron performed best under water stress condition. So, in the region, where the irrigation water is a matter of concern in case of cost and availability, the variety Aloron may be a better option to cultivate as it provides equal yield as mega variety BRRI dhan 29 under no water stress condition. However, further experimentation will need to be executed in different agro-ecological zones with more varieties under water stress condition to reach a specific conclusion and recommendation.

\section{References}

Anonymous. 1988. The Year Book of Production. FAO, Rome, Italy.

BBS (Bangladesh Bureau of Statistics). 2020. Statistical Yearbook of Bangladesh for 2020. Bureau of Statistics, Statistics and Informatics Division, Ministry of Planning, Government of the People's Republic of Bangladesh, Dhaka, Bangladesh. 
Caine, R.S., X. Yin, J. Sloan, E.L. Harrison, U. Mohammed, T. Fulton, A.K. Biswal, J. Dionora, C.C. Chater, R.A. Coe, A. Bandyopadhyay, E.H. Murchie, R. Swarup, W.P. Quick andJ.E. Gray.2019.Rice with reduced stomatal density conserves water and has improved drought tolerance under future climate conditions. New Phytol. 221(1): 371-384.

Castilo, E, J. Siopongco, R.J. Buresh, K.T. Ingram and S.K.D. Datta. 1987. Effect of nitrogen timing and water deficit on nitrogen dynamics and growth of lowland rice. IRRI Saturday, 7 November 1987. Los Banos, Laguna, Phillippines.

Chowdhury, M.J., M.T. Islam, and M.O. Islam. 2004. Evaluation of two local Transplant aman rice cultivars and a mutant under soil moisture stress. Bangladesh J. Soc. Agril.Sci. Technol.1(1\&2): 127-131.

Fofana, M., M. Cherif, B. Kone, K. Futakuchi and A. Audebert. 2010. Effect of water deficit at grain repining stage on rice grain quality. J. Agric. Biotech. Sustain. Dev. 2(6): 100-107.

FRG. 2012. Fertilizer Recommendation Guide. Bangladesh Agricultural Research Council (BARC). Farmgate, Dhaka 1215. p.74.

Gardner, F.P., R.B. Pearce and R.L. Mistechell. 1985. Physiology of Crop Plants. Iowa State Univ. Press, Powa. p.66.

Halder, K.P., M.S. Islam, M.R. Manir and M.A. Ali. 2018. Moisture Stress and Different Rates of Nutrients on Growth and Yield of Rice. Bangladesh Rice J. 22(2): 23-30.

Halder, K.P. and S.W. Burrage. 2003. Drought stress effects on water relations of rice grown in nutrient film technique. Pak.J.Biol.Sci. 6 (5): 441-444.

Keller, M. 2005. Deficit irrigation and vine mineral nutrition. American J. Enol. Vitic. 56: 267-283.

Maneepitak, S., H. Ullah, A. Datta, R. Shrestha, S. Shrestha and B. Kachenchart. 2019. Effects of water and rice straw management practices on water savings and greenhouse gas emissions from a doublerice paddy field in the Central Plain of Thailand. European J. Agron. 107:18-29.

Mannan M.A., M.S.U. Bhuiya, M.I.M. Akhand and M.M. Zaman. 2012. Growth and yield of basmati and traditional aromatic rice as influenced by water stress and nitrogen level. J. Sci. Found.10(2):5262.

Molden, D., T. Oweis, P. Steduto, P. Bindraban, M.A. Hanjra and J. Kijne. 2010. Improving agricultural water productivity: Between optimism and caution. Agric. Water Mngt. 97: 528-535.

Mostajeran, A. and V. Rahimi-Eichi. 2009. Effects of drought stress on growth and yield of rice (Oryza sativa L.) cultivarsand accumulation of proline and soluble sugars in sheathand blades of their different ages leaves. American-Eurasian J. Agric. Environ. Sci. 5(2): 264-272.

Murshida, S., M.R. Uddin, M.P. Anwar, U.K. Sarker, M.M. Islam and M.M.I. Haque. 2017. Effect of variety and water management on the growth and yield of boro rice. Progress. Agric.28(1): 26-35.

Sabetfar, S., M. Ashouri, E. Amiri and S. Babazadeh. 2013. Effect of drought stress at different growth stages on yield and yield component of rice. Persian Gulf Crop Protect. 2(2): 14-18.

Sarvestani,Z.T., H. Pirdashti, S.A.M.M. Sanavy and H. Balouchi . 2008. Study of water stress effects in different growth stages on yield and yield components of different rice (Oryza sativa L.) cultivars. Pak. J. Biol. Sci. 11(10):1303-1309.

Sokoto, M.B. and A. Muhammad. 2014. Response of rice varieties to water stress in Sokoto, Sudan Savannah, Nigeria. J. Biosc. Medicines. 2: 68-74.

Waraich, E.A., R. Ahmad, Saifullah, M.Y. Ashraf and Ehsanullah. 2011. Role of mineral nutrition in alleviation of drought stress in plants. Australian J. Crop Sci. 5: 764-777.

Zubaer, M.A., A.K.M.M.B. Chowdhury, M.Z. Islam, T. Ahmed and M.A. Hasan. 2007. Effects of water stress on growth and yield attributes of aman rice genotypes. Intl. J. Sustain. Crop Prod. 2(6): 2530. 